\title{
Wind erosion of sand placed inside a rectangular box
}

\author{
Almerindo D. Ferreira *, Rui A. Oliveira \\ Department of Mechanical Engineering, University of Coimbra, Polo II, 3030-788 Coimbra, Portugal
}

\section{A R T I C L E I N F O}

\section{Article history:}

Received 17 May 2007

Received in revised form

4 September 2008

Accepted 19 September 2008

Available online 6 November 2008

Keywords:

Wind erosion

Free-surface change

Sand erosion

Wind dispersion

Wind tunnel

Aeolian erosion

\begin{abstract}
A B S T R A C T
This paper shows some experimental results on wind erosion of sand particles, placed inside a rectangular box with the upper surface exposed to the flow. Data presented were achieved from a wind tunnel simulation. Results include erosion rate, free-surface profile change, vertical deformation rate, and also flow topology for several loose boundary geometries. Some visualization tests illustrate the path of particles, depicturing how the eroded bed evolutes.

The main objective of this work is focused on the achievement of data useful for the benchmark of numerical models dealing with wind erosion phenomenon, in situations where the free-surface changes due to the removal and/or deposition of granular particles. Practical cases related with the present work include situations where coal particles are removed from stockyard piles, or dust is released during transportation of a granular material in open containers.
\end{abstract}

(c) 2008 Published by Elsevier Ltd.

\section{Introduction}

It is common to find in nature situations where aeolian erosion of solid particles occurs. Such natural situations include wind exposed coal piles in stockyards, drifting of sand or snow, or even other granular materials stocked or transported in open containers.

Those materials, once exposed to air flow, are subject to the erosion process and, consequently, to the movement of particles through the ejection, suspension, rolling, creeping, impact and rebounding phenomena (Bagnold, 1941). As a result of such particles' movement, namely deposition and entrainment, the bed's loose-surface is modified over time.

Numerous studies have been devoted to the problem of erosion of a granular material laying on a flat bed (e.g. Owen, 1964; Nalpanis et al., 1993; Nishimura and Hunt, 2000; Liu et al., 2006), most of them relating flow properties with the erosion rate, and admitting maintenance of flat bed geometry. On the contrary, Beyers et al. (2004), using a CFD code, simulated the transient three-dimensional turbulent flow of snow drifting around a cube obstacle, considering both the entrainment and accumulation, and the consequent bed-layer modification.

Considerable research work has also been done on the evaluation of fugitive dust control devices, like compact barriers or windbreak porous fences (Badr and Harion, 2005; Lee et al., 2002), although without considering changes of storage pile's loose-surface due to erosion.

Research on wind-induced releases from storage tanks usually deals with liquid or gas substances (Hort and Robins, 2002), while

\footnotetext{
* Corresponding author. Tel.: +351239790 732; fax: +351239790771.

E-mail address: almerindo.ferreira@dem.uc.pt (A.D. Ferreira).
}

the wind erosion of granular materials placed inside containers is scarcely studied, despite its frequent occurrence for example in transport systems like trucks or train wagons. Mansour (2004) computed the wind flow, for several train-wagons' cover geometries, aiming its relation with erosion rates, but no deformation of the loose-surface was considered.

The fugitive dust, both during transportation or from stock piles, is susceptible to creating environmental impact, since the dispersion of pollutants might affect communities, landscape, or even create agricultural concerns (e.g. López, 1998) or, on the other hand, economical loss of valuable materials. Among other possible examples, dust released from the transportation of granular materials in open railway cars is highly susceptible to create human impact due to the deposition of dust along the railway path passing through or nearby communities, but also to produce economic loss due to the waste of granular material (Ferreira et al., 2003).

This investigation presents some results obtained after a wind tunnel simulation where sand particles are wind-eroded from inside a rectangular box, with the upper surface exposed to the flow. Issues discussed include lost time-rate, bed free-surface change in time and space, and flow topology modification due to the change of free-surface shape. These data are valuable for the development and benchmark of related computational models ( $\mathrm{Ji}$ et al., 2004), which might be useful for environmental impact studies related to dispersion of solid pollutants.

\section{Experimental setup}

This section provides general information about the wind tunnel, box model geometry, instrumental setup, and sand granulometry. 


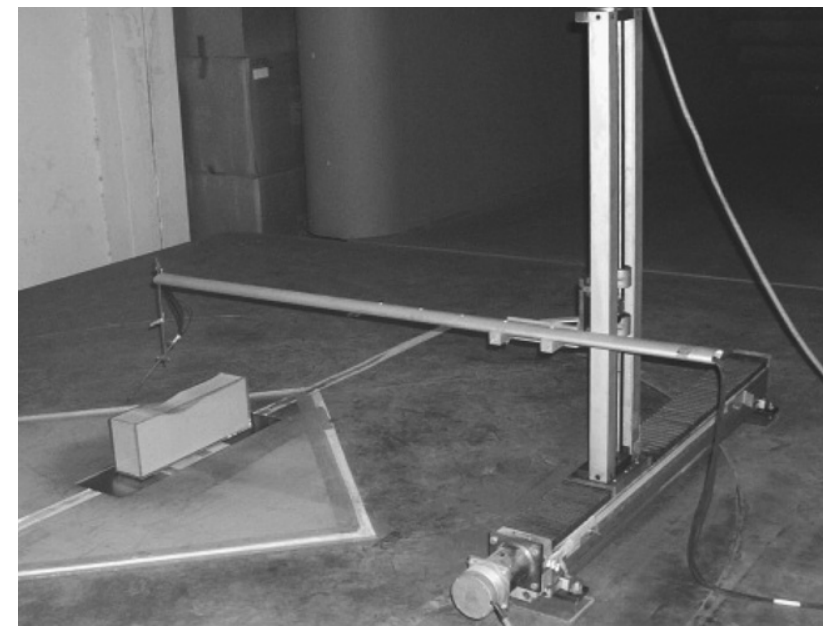

Fig. 1. Perspex box with sand placed over the scale in the wind tunnel chamber; transverse system with the seven-hole probe mounted.

\subsection{Wind tunnel}

The wind tunnel used for this study is installed at the Industrial Aerodynamics Laboratory (LAI), which is part of the ADAI facilities (University of Coimbra-Portugal). It has an open working chamber, $5 \mathrm{~m}$ long and $2 \mathrm{~m}$ wide. For the experiments the undisturbed velocity $\left(U_{0}\right)$ was chosen equal to $11.4 \pm 0.3 \mathrm{~m} / \mathrm{s}$, measured at the entrance of the working chamber.

An electronic scale Kern 700, with a precision of $\pm 0.05 \mathrm{~g}$, connected to a personal computer, was used to measure the erosion rate of sand. Although the sample rate for the weight measurements was set to $1 \mathrm{~Hz}$, due to the flow turbulence and the consequent fluctuations on the registered weight, mean values of 50 samples were considered for the analysis, as it will be shown in a later section. The scale, covered with a glass levelled with the working chamber base to reduce its interference with the flow, was positioned $2.4 \mathrm{~m}$ downstream the nozzle's edge and the parallelepiped box over the glass (Fig. 1). No vorticity generators or boundary layer thickness elements were used to change the velocity profile in the working section. The profiles of the streamwise velocity component $(u)$ and turbulence intensity $(i)$, measured at the upstream edge of the glass when the working chamber was empty, vary according to Fig. 2. Concisely, the velocity profile can be properly fitted by a power law $u / U_{0}=$ $(z / \delta)^{\alpha}$, with the boundary layer thickness $(\delta)$ equal to $0.4 \mathrm{~m}$ and $\alpha=0.11$. The turbulence intensity $(i)$ remains nearly unchanged with height, and it can be assumed equal to $15 \%$.

\subsection{Model geometry}

The box, which was made of perspex to enable photo and video recordings of the free-surface shape, was placed over the glass and scale, close to the centre of the wind tunnel working chamber, with the longer faces aligned with the main flow. The size and geometry of the box (Fig. 3) was chosen based on previous studies using a train model (Ferreira and Vaz, 2004).

\subsection{Sand granulometry}

Relatively fine sand was adopted as a granular material. The granulometry of the sifted sand used in the tests, has the particle size distribution shown in Fig. 4, where it can be seen that the prevailing grains have a diameter of approximately $0.5 \mathrm{~mm}$. The sand density, measured for this specific granulometry, is equal

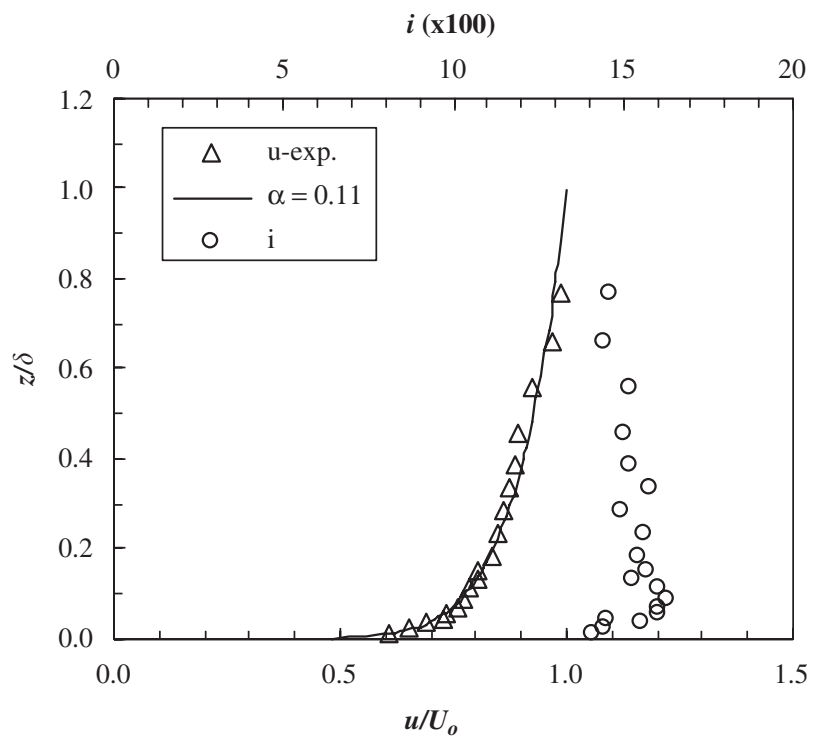

Fig. 2. Streamwise velocity component and turbulence intensity profiles, measured at the centre of the wind tunnel working chamber.

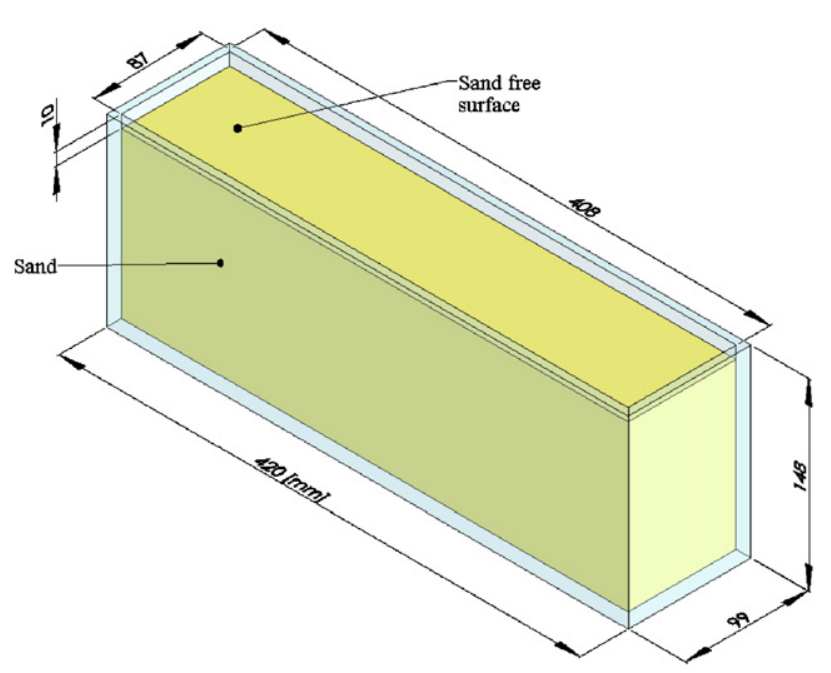

Fig. 3. Geometry and dimensions of the parallelepiped box used in the experiments.

to $1497 \mathrm{~kg} / \mathrm{m}^{3}$. Prior to testing, the sand was dried, and no cohesive forces between grains were observed.

For all the tests, as illustrated in Fig. 3, the free-surface, at the onset of each experiment, was set horizontal, flat, and placed $10 \mathrm{~mm}$ below the box's upper edges.

\section{Results and discussion}

\subsection{Repeatability of results}

The wind flow around a blunt body is highly complex, usually characterized by vortex shedding and strong streamline curvature. As frequently pointed out, high levels of vorticity and turbulence are observed in such kind of flow situations. Being so, as the erosion process is strongly affected by turbulence, and due to the random nature of the vortex shedding and buffeting, it is important to analyze the repeatability level of the final result achieved. For this purpose, three preliminary tests (A, B and C) were performed for the same initial boundary conditions. 


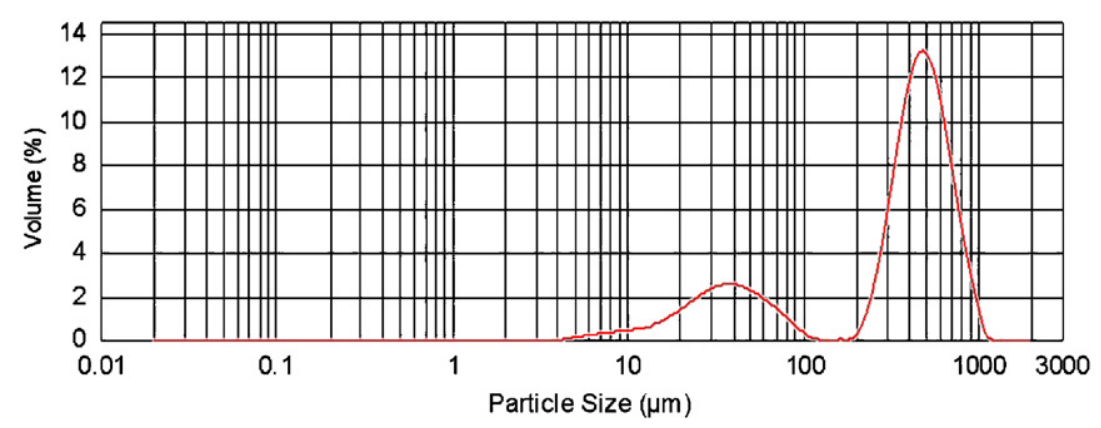

Fig. 4. Granulometry of the sand used in the experiments.

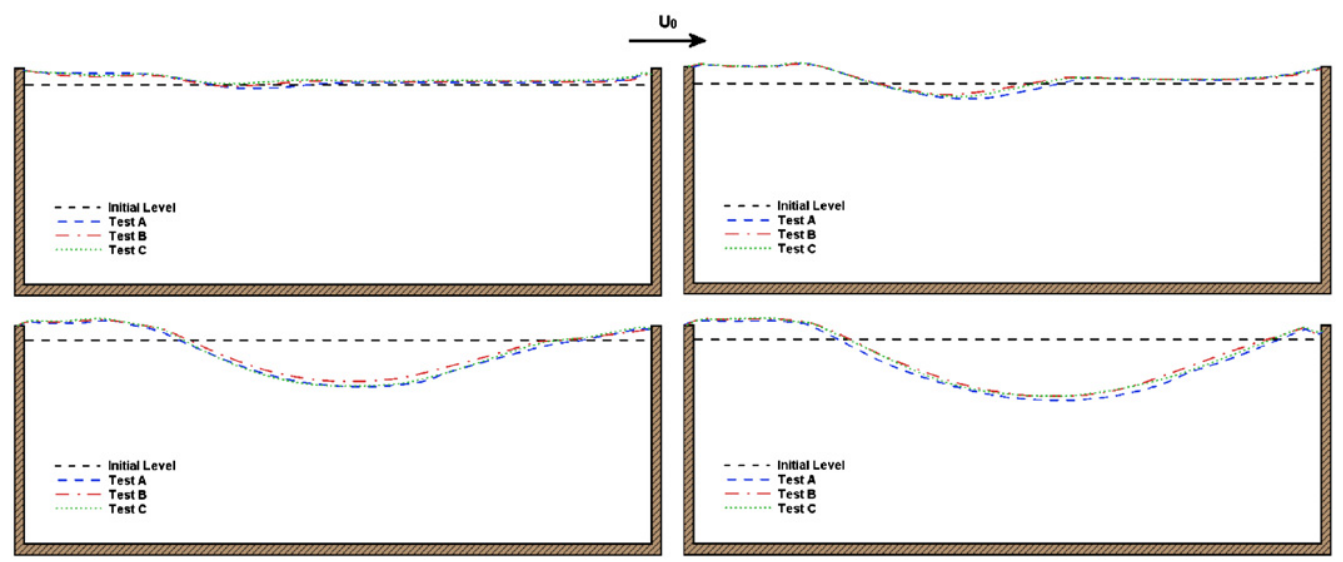

Fig. 5. Free-surface profile, along the central plane, for $t=4,10,60$ and $120 \mathrm{~min}$.

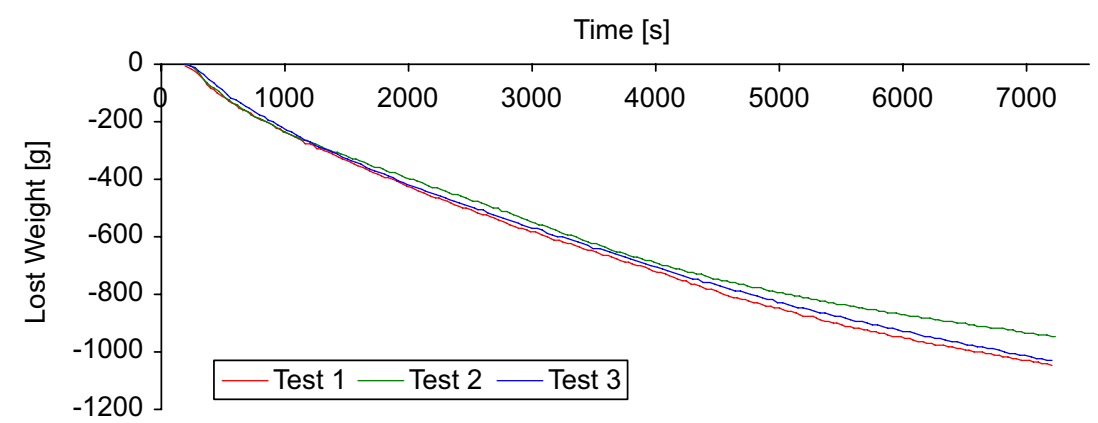

Fig. 6. Time evolution of the total amount of sand released (in grams), observed in three similar experiments.

Analysis of the repeatability was based on the shape of the granular free-surface bed at various time steps, that is, the timelength of the erosion process measured from the beginning of the box's wind-exposition. Several photos of the free-surface profile were registered at different time instances $(t)$, starting at $t=4 \mathrm{~min}$, and culminating at $t=120 \mathrm{~min}$. The three recorded profiles, for some of the time-steps, are presented in Fig. 5. Those results reveal a high level of repeatability, despite some discrepancies observed primarily at the central region of the box and for the largest time step $(t=120 \mathrm{~min})$. This conclusion supports the validity of the results hereafter presented, giving them credibility when used for the benchmark of computational models.

\subsection{Erosion rate of sand released from inside the box}

During the experiments it was verified that the flow induces a lift force on the scale and/or model. The lift was measured for different shapes of the free-surface, and it can be said that its magnitude ranges between $63.6 \mathrm{~g}$ (for $t=0$ ) and $68 \mathrm{~g}$ (for $t>60 \mathrm{~min}$ ). Results in Figs. 6 and 7 show the net loss, i.e. with the lift force deducted. All the curves were computed based on mean values of 50 samples.

Fig. 6 illustrates the time evolution of the cumulative amount of sand released from the box (in grams), observed for three similar experiments. The similarity between the results of tests 1 and 3 is remarkable, while curve of test 2 differs for time instants exceeding $4000 \mathrm{~s}$.

For the same data set, Fig. 7 depicts the erosion rate per unit of area. The maximum occurs in the initial stage, when the box is still almost filled. Then, also, the free-surface is flat, and somehow the loss rate can be compared with the situation of a flat bed. Bagnold (1941) suggests the following formula for the total horizontal flux, including creep and saltation:

$q=C \sqrt{\frac{d}{D}} \frac{\rho}{g} u_{*}^{3}$ 
where $C=1.5$ for nearly uniform sand, $D$ is the grain diameter of a standard $0.25 \mathrm{~mm}$ sand, $\rho$ the air density, $g$ the acceleration of gravity, and $u^{*}$ the threshold friction velocity. Measurements using sand with $d=0.5 \mathrm{~mm}$ and for the flat bed case, led to $u^{*}=0.40 \mathrm{~m} / \mathrm{s}$. Using these values in Eq. (1), $q=16.3 \mathrm{~g} /\left(\mathrm{m}^{2} \mathrm{~s}\right)$, which compares quite well with the maximum values measured

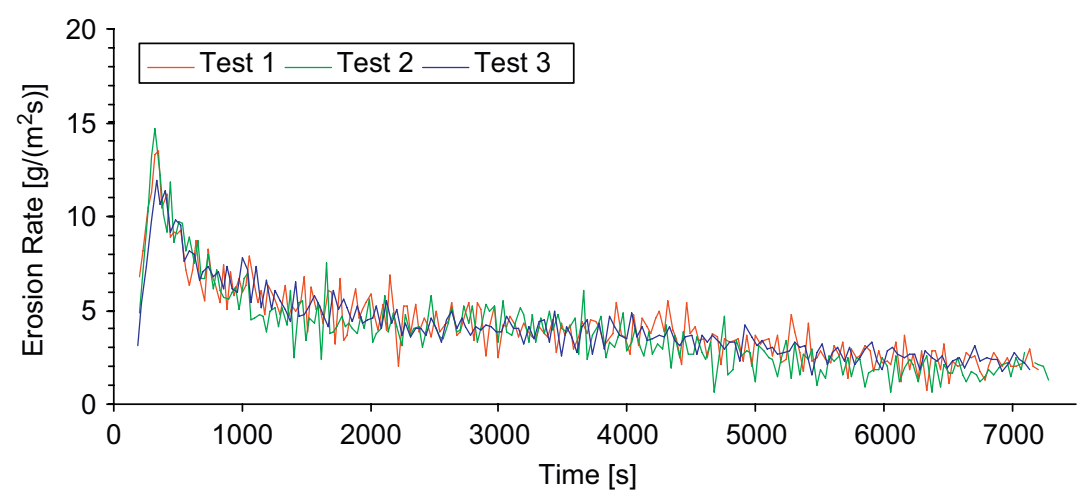

Fig. 7. Time evolution of the erosion rate, per unit area, computed for three similar experiments.

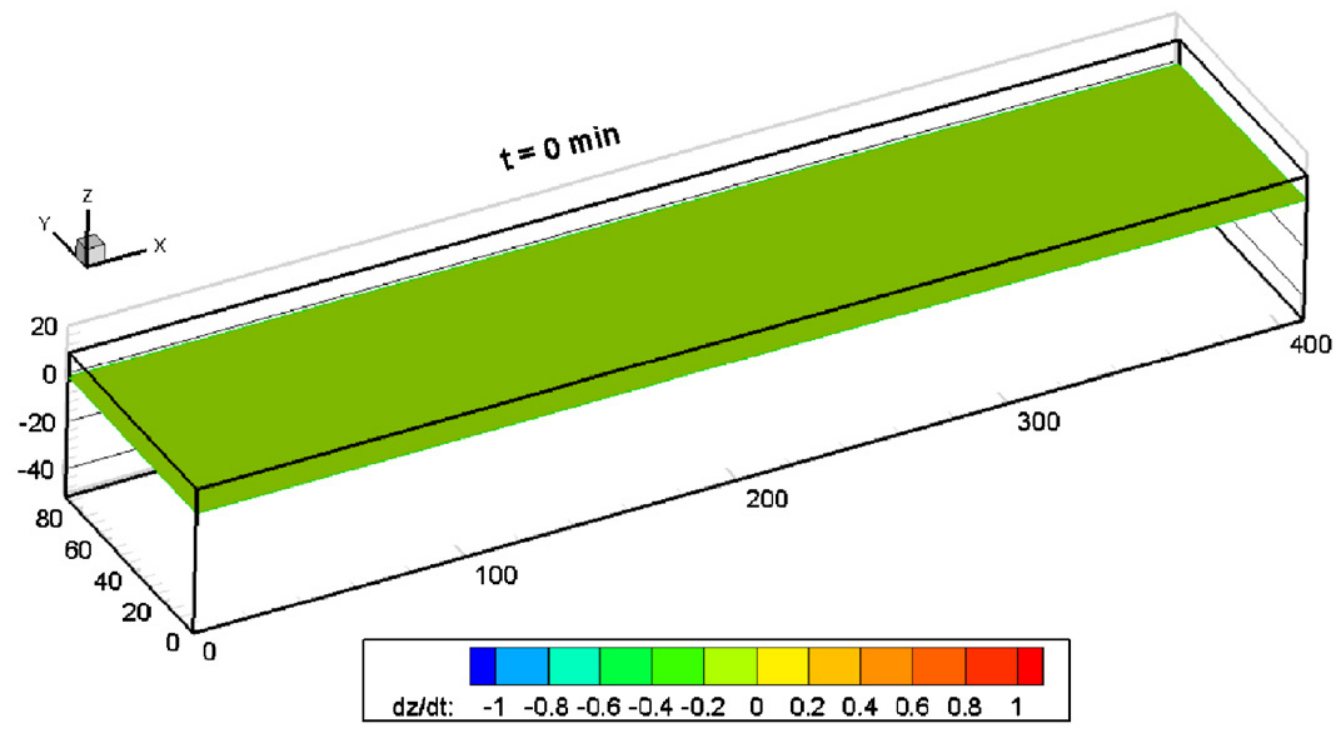

Fig. 8. Free-surface shape for $t=0 \mathrm{~min}$.

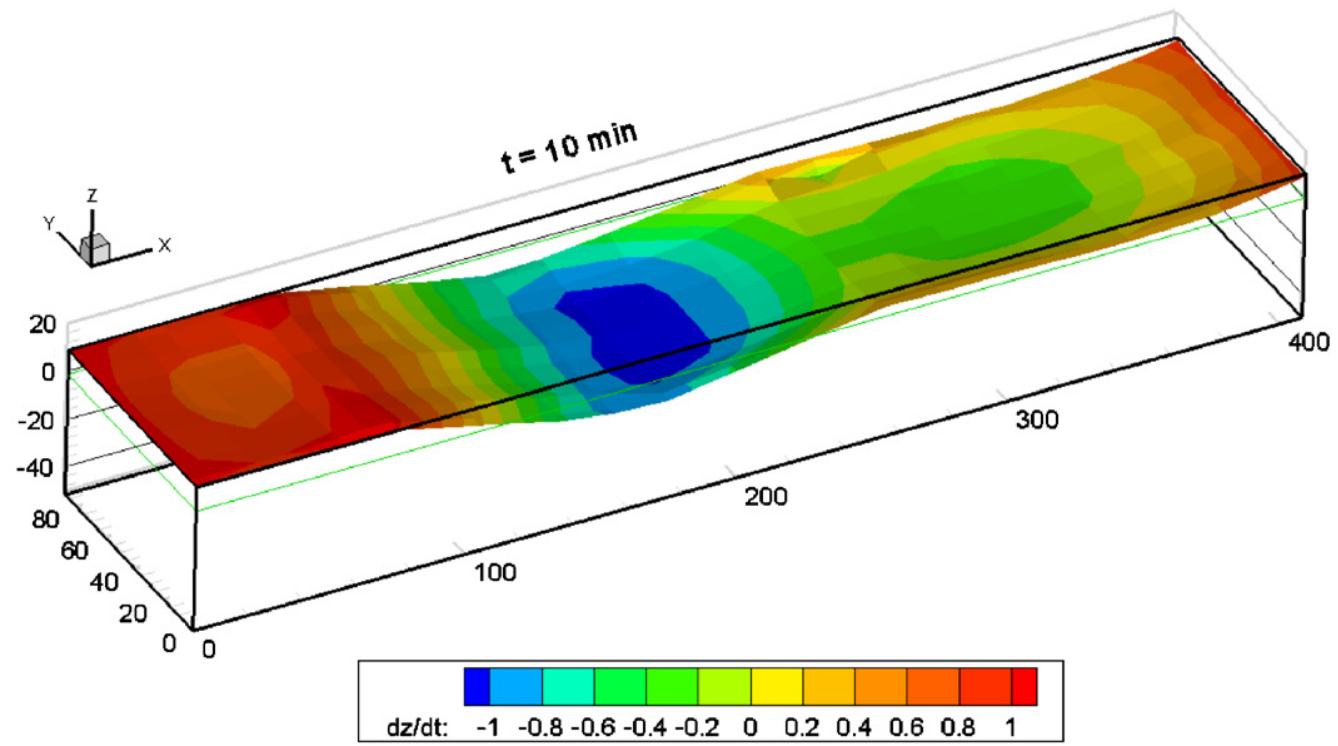

Fig. 9. Free-surface shape and vertical displacement rate for $t=10 \mathrm{~min}$. 
at the start of all tests-the average of the three peak values is $13.4 \mathrm{~g} /\left(\mathrm{m}^{2} \mathrm{~s}\right)$.

After the initial stage the free-surface changes rapidly, being no longer flat, and the flux decreases gradually tending to $2 \mathrm{~g} /\left(\mathrm{m}^{2} \mathrm{~s}\right)$ for $t>5000 \mathrm{~s}$, which is nearly one order of magnitude smaller comparatively to the flat bed case.

\subsection{Three-dimensional time-change of the free-surface}

Figs. 8-14 show the deformation of the free-surface, for several time-instants, starting at $t=0 \mathrm{~min}$ when the granular bed is flat and placed $10 \mathrm{~mm}$ bellow the top edges of the box, as mentioned. For the different time steps analyzed, the free-surface coloration is related to the local "vertical displacement rate" $\partial z / \partial t\left(t_{i}\right)$, calculated for the instant $t_{i}$ through backward differences using values from instants $t_{i-1}$ and $t_{i}$. The units in those figures are $[\mathrm{mm} /$ minute], defined as positive when the surface moves upward, in regions where the deposition of sand surpasses the erosion. Clearly, in zones where the free-surface moves deeper, the erosion locally surpasses the accumulation phenomenon.

For every result shown in Figs. 9-14, each corresponding erosion experiment was consistently started from $t=0$, which means that the same initial free-surface level was respected, after the perspex box had been refilled with sand. The duration of each experiment was set according to the desired time step. At the end, the free-surface was made rigid ("frozen") using regular hairspray. After a drying period, the depth of the free-surface was measured at numerous discrete locations, based on a previously defined horizontal grid covering the entire upper box surface.

In Fig. 9, it can be seen that during the first 10 min the larger modifications occur near the upwind vertical box wall, making evident the movement of sand from the centre of the box to the upward region, filling the box up entirely in that area. Curiously, it was observed that the surface, after this first period of $10 \mathrm{~min}$, remained nearly unchanged, being the free bed-surface effectively located a few millimeters above the top of the perspex box.

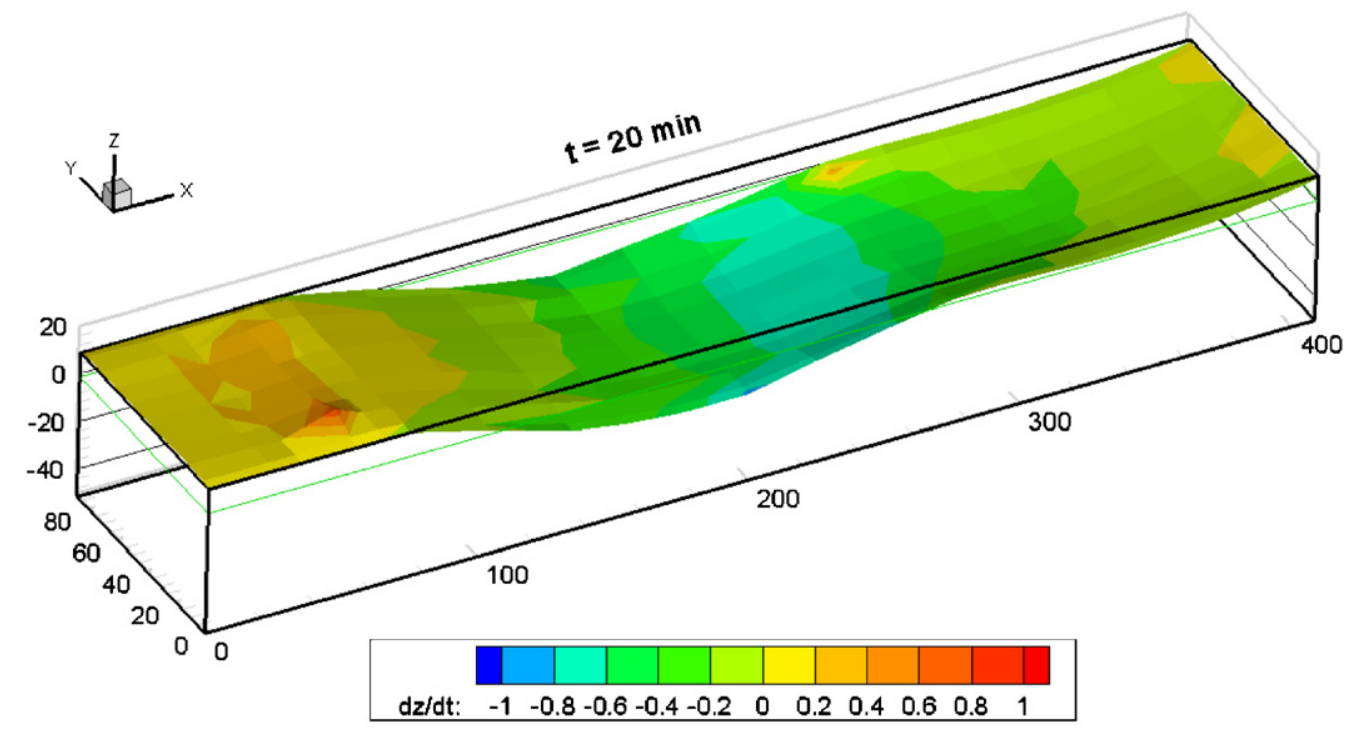

Fig. 10. Free-surface shape and vertical displacement rate for $t=20 \mathrm{~min}$.

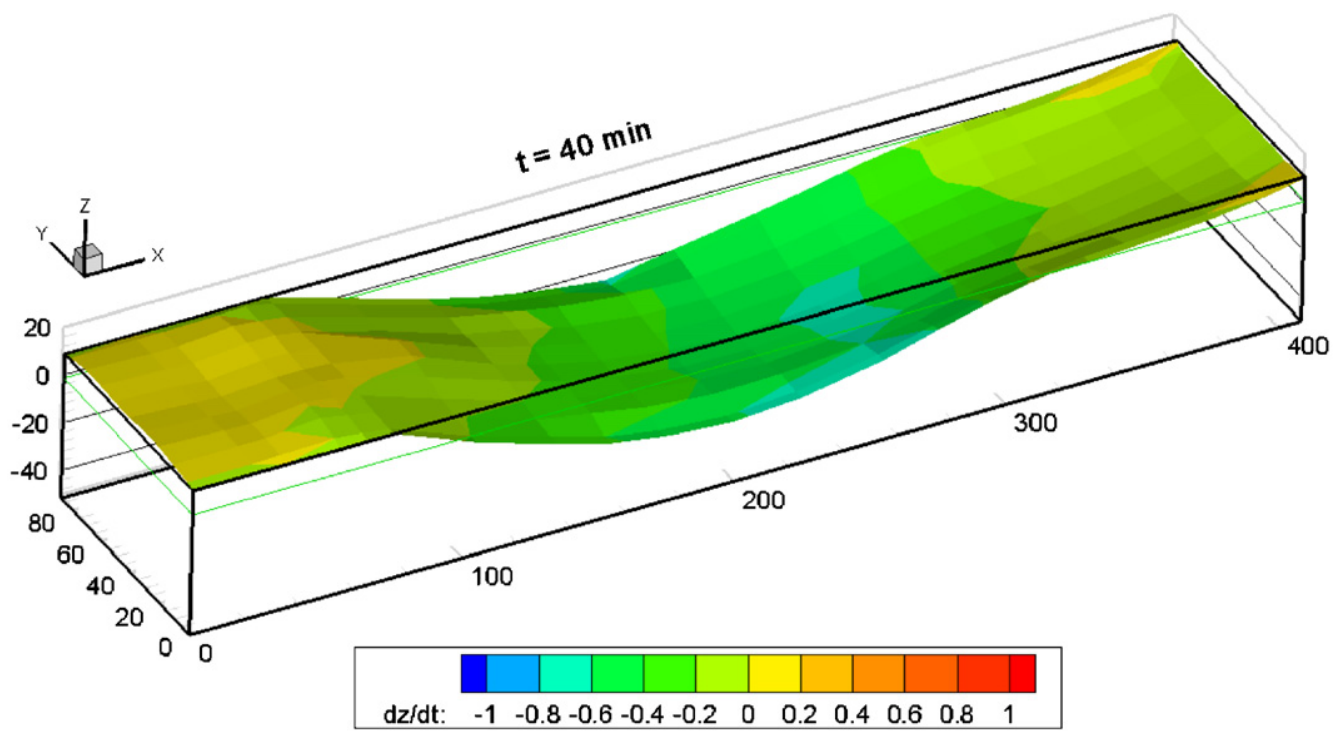

Fig. 11. Free-surface shape and vertical displacement rate for $t=40 \mathrm{~min}$. 


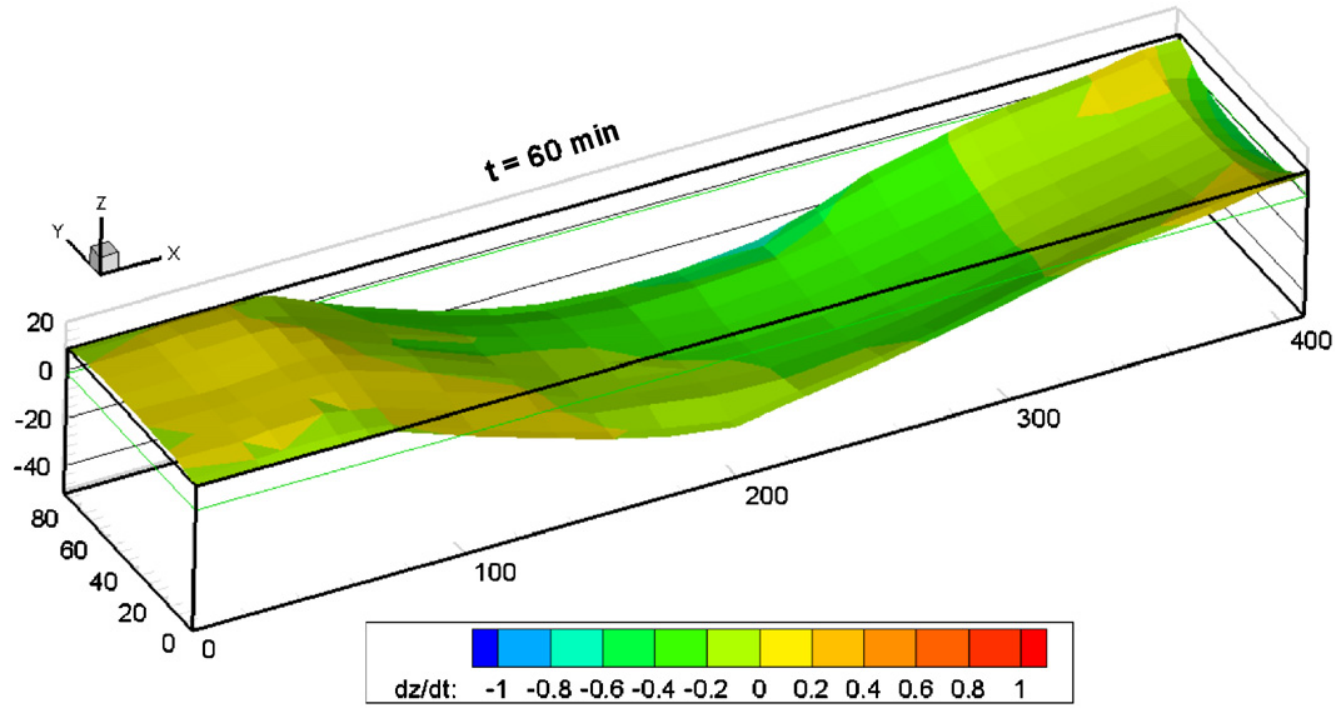

Fig. 12. Free-surface shape and vertical displacement rate for $t=60 \mathrm{~min}$.

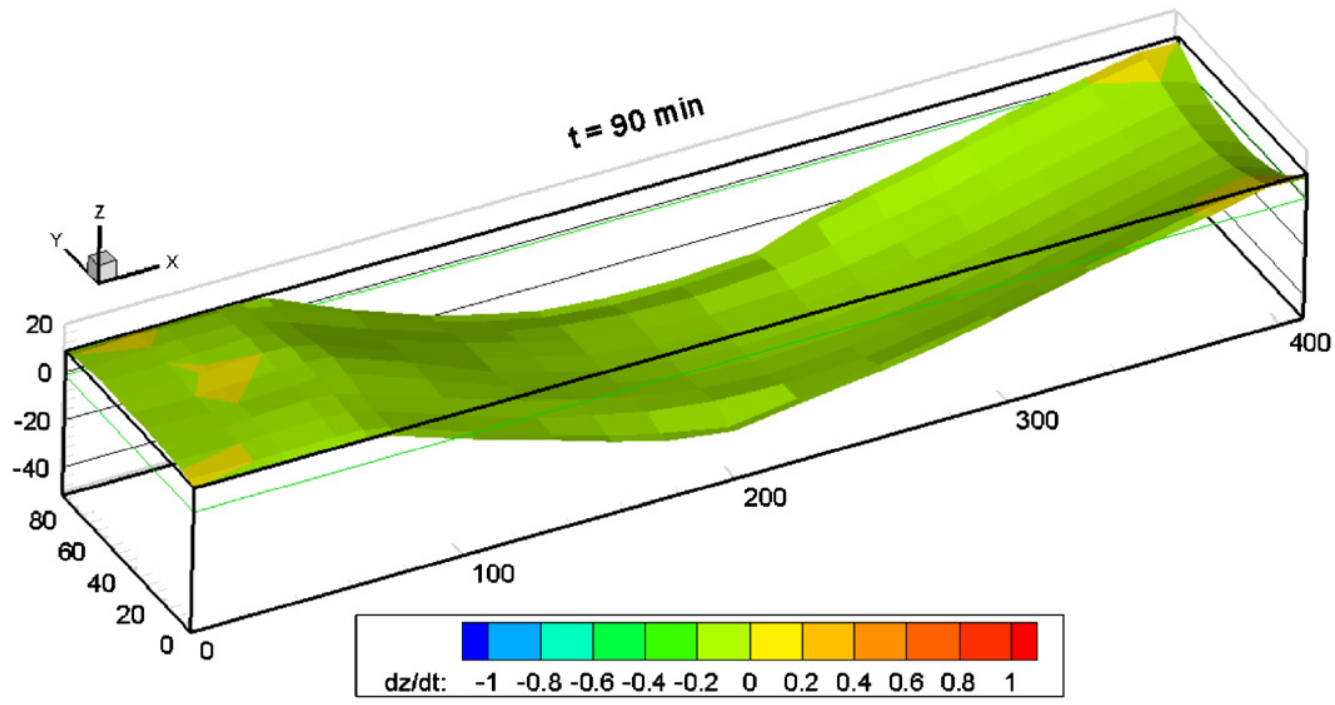

Fig. 13. Free-surface shape and vertical displacement rate for $t=90 \mathrm{~min}$.

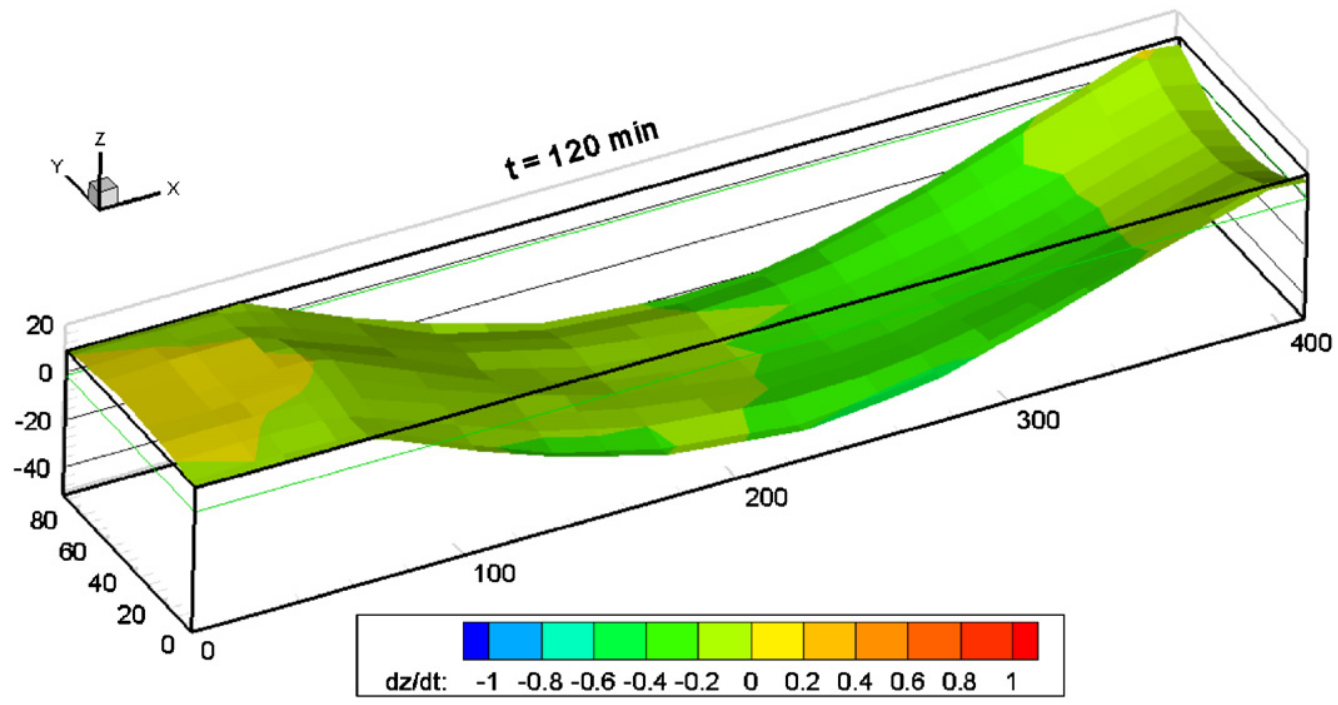

Fig. 14. Free-surface shape and vertical displacement rate for $t=120 \mathrm{~min}$. 
Near the downwind extreme of the box, accumulation of sand also occurs, although with a weaker intensity comparatively to what happens in the upward region. Initially a ramp is formed there, which, as visually observed, is used for the escape of particles moving mainly through the creeping process. After $t=40 \mathrm{~min}$, this downward ramp shape changes, as a depression close to the downwind surface appears, which is clearly visible at $t=60$ min (Fig. 12). This surface depression moves deeper for later instants, likely as a consequence of the development of a transverse turbulent vortex nearly parallel with the $y$-axis, possibly induced by the recirculation zone present in the leeward end of the box.

As shown below, after the depression surface is created, the release of sand through that end still persists, as a result of the particles ejected from the box and uplifted after the final edge of the formed ramp.

\subsection{Wind-flow profiles for different free-surface geometries}

For the wind flow characterization a seven-hole pressure probe (Silva et al., 2003) was used. The free-surface nearest locations for the measurements were defined after the determination of the surface profile, as previously shown. Fig. 15 illustrates a typical example of the grid adopted for the measurements, with all points set in the central $x-z$ plane, coincident with the longitudinal box's symmetry plane.

Some results are shown in the form of vector plots in Figs. 16-19. Due to restrictions of the probe used, regarding its unsuitability for measurements in reversing flow zones, some of the selected grip points show no vector. Nevertheless, it is still useful as it clarifies the existence and extension of the recirculation area.
The flow topology is mainly imposed by the blunt geometry of the box due to its sharp edges. Nevertheless, despite undetectable changes in the general flow circulation, some evident changes are observed near the sand free-surface, especially in the final quarter-length sector. There, the analysis of flow vectors confirm the results discussed in the previous section, and facilitate the definition of general movements of sand along the free-bed as shown in Fig. 20.

In fact, from the video analysis, and its relation to the velocity profiles, three main zones can be distinguished inside the box, and even a fourth one during the last time instants. Most of the sand accumulated in zone 1 is dragged from zone 2 due to the recirculation zone near the leading edge. As the velocity in the vicinity of the surface in zone 1 is not high enough, sand displaced from zone 2 is deposited in the upwind zone. The upwind box edge implies the formation of the large vortex area over the box, being the recirculation basically unaffected by the shape of the granular-bed, which explains the nearly steady shape of the freesurface in zone 1 after local deposition has settled down.

Some sand is also moved downstream from zone 2, referred here as the erosion zone as it corresponds to the area where the process occurs more intensely, to zone 3 helping the formation of the ramp there. Zone 4 is characterized by the appearance of a depression zone across its entire width, which occurs only in a later stage, as previously mentioned.

In order to visualize the movement of sand particles away from the box, a laser light sheet was used for image recording. This sheet was in the plane assumed for the flow field measurements, being then coincident with the longitudinal box symmetry plane.

Figs. 21 and 22 show the release of particles through the upwind and downwind edges of the box. Particularly in the first

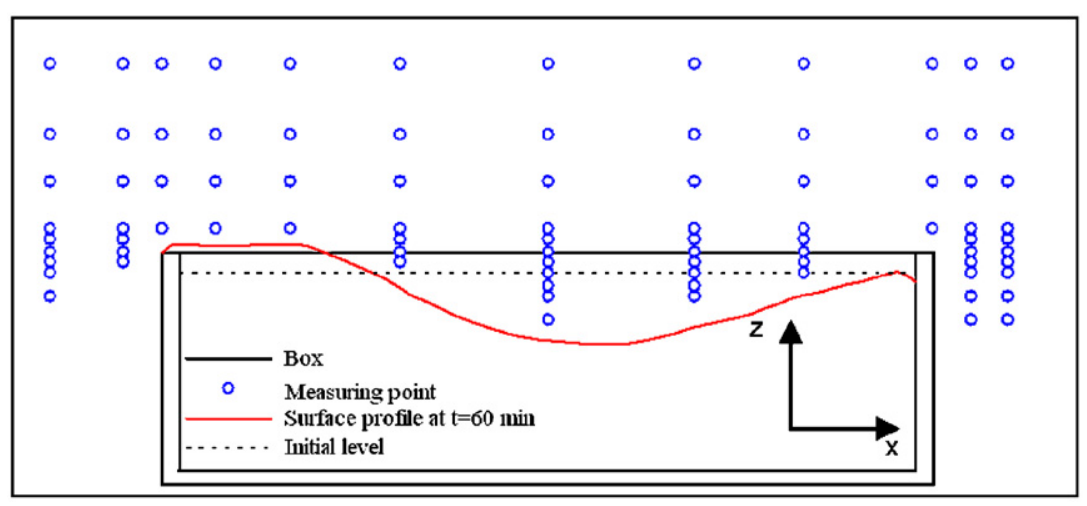

Fig. 15. Free-surface shape at $t=60 \mathrm{~min}$ and grid points selected for the measurement of the mean velocity-components.

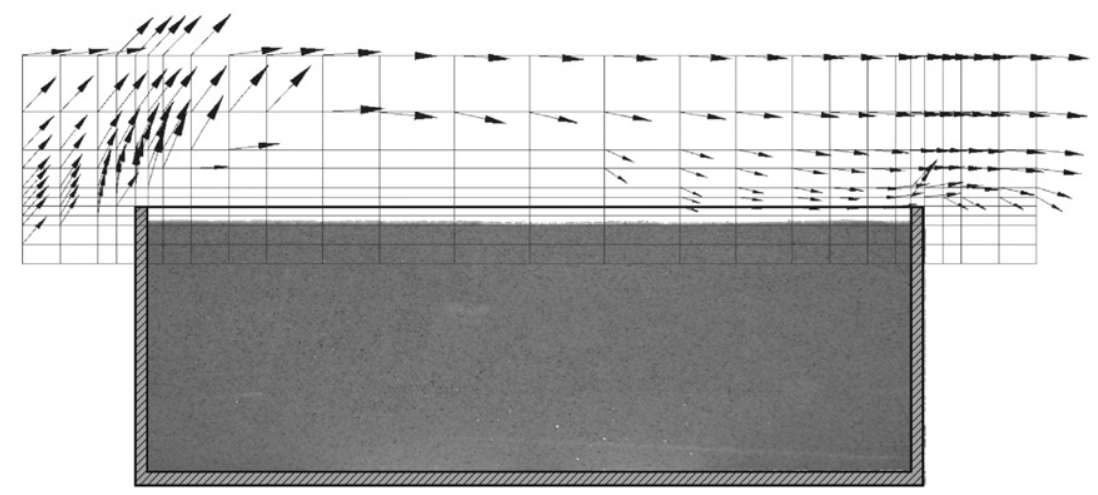

Fig. 16. Flow field measured at the central plane for $t=0 \mathrm{~min}$. 


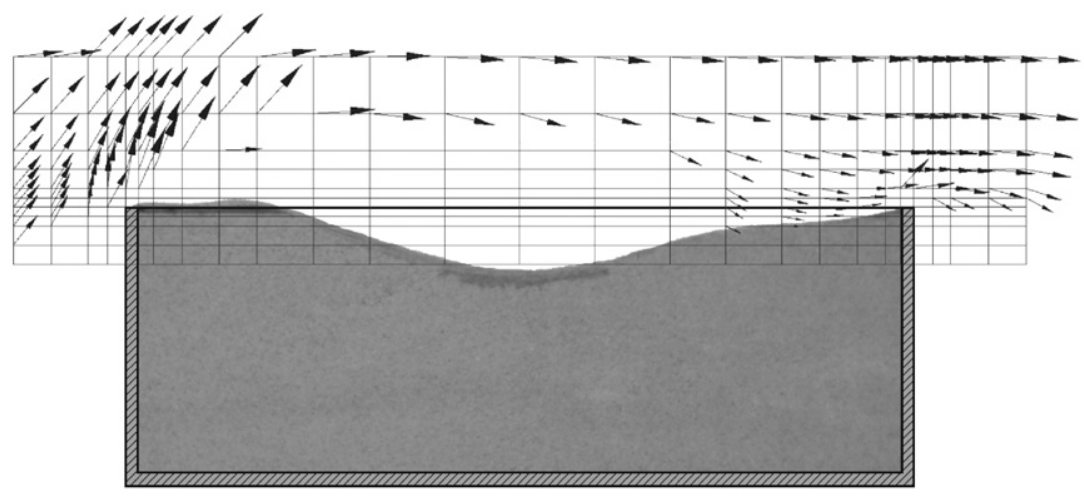

Fig. 17. Flow field measured at the central plane for $t=40 \mathrm{~min}$.

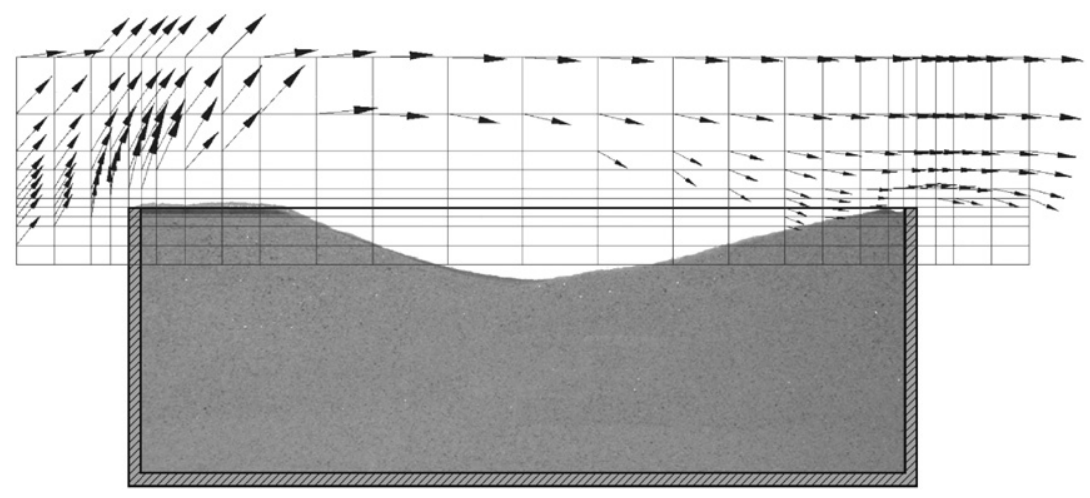

Fig. 18. Flow field measured at the central plane for $t=90 \mathrm{~min}$.

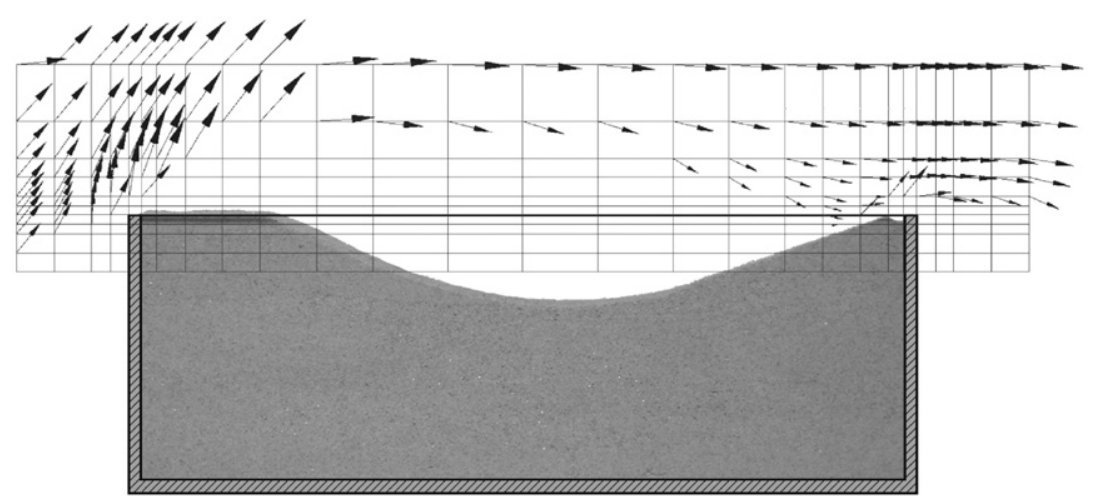

Fig. 19. Flow field measured at the central plane for $t=120 \mathrm{~min}$.

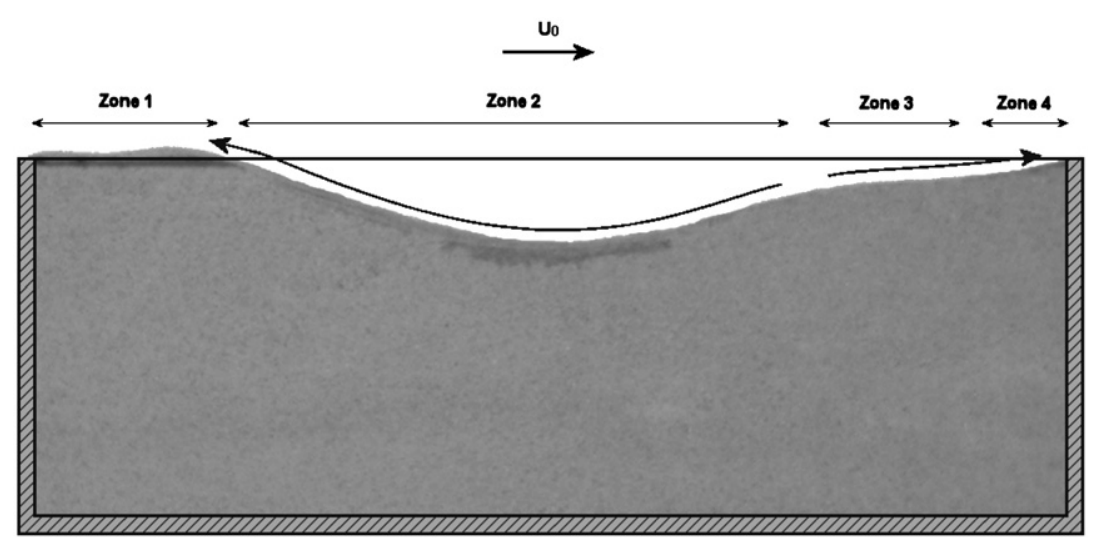

Fig. 20. Observed movement of the sand inside of the box due to aeolian erosion 

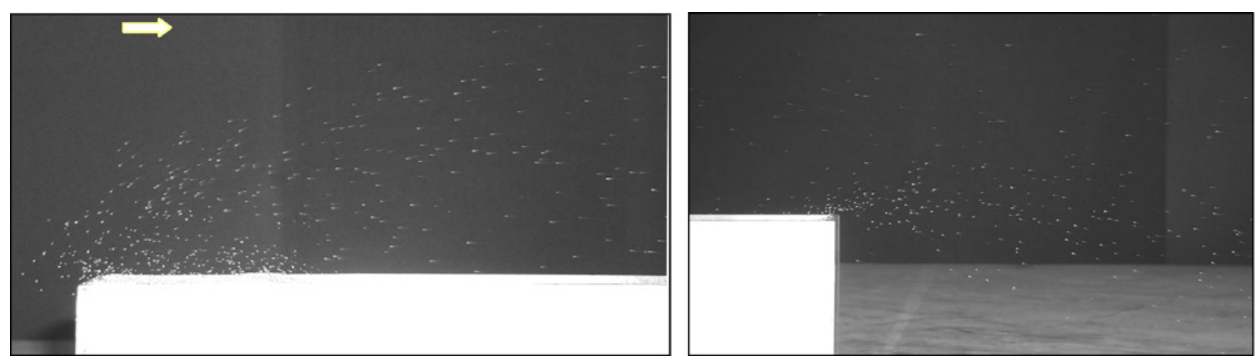

Fig. 21. Observed movement of sand near the leading (left) and trailing (right) box's edges.

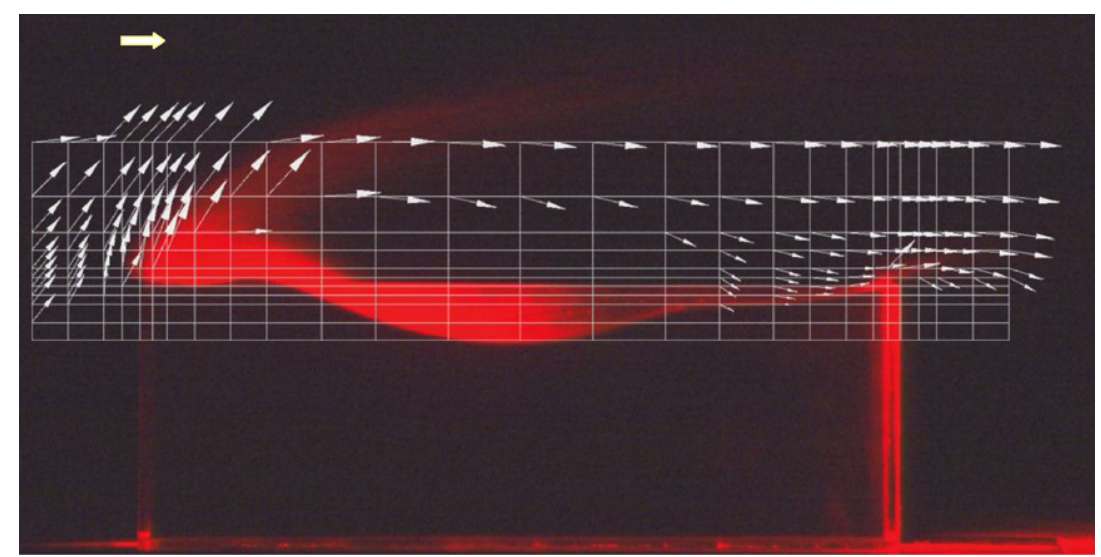

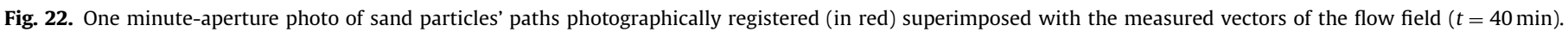

mentioned location (Fig. 21, left), the picture revealed very interesting observations as some particles released clearly move to a position placed considerably upwind the leading face, and are just then lifted-up. Most of those particles are then carried by the bulk flow, but some of them return to the box, disturbing others due to their impact on the free-surface, intensifying the erosion process mainly in zone 2 .

Also from the observations made available with the laser experiments, Fig. 22 shows the movement of sand particles in the vicinity of the box. The paths of many grains are marked in red, registered photographically during one-minute aperture of the lens. The photo is superimposed with the experimental flow field, measured as described before. Both sets of results were observed in the symmetry plane and for $t=40 \mathrm{~min}$. From this figure it is clearly evident that the release of particles from inside the box occurs mainly through the leading edge, which is in harmony with what had been discussed about Fig. 20.

\section{Conclusions}

Experimental results were presented for a situation where a granular material, placed inside of a parallelepiped box, is eroded due to the wind flow. Data presents the evolution, in time, of the erosion rate, of the flow field in the central plane, and of the eroded-surface three-dimensional shape. The experiments, despite the expected intense-turbulence of the flow, showed a high level of repeatability. From the flow-field measurements it was concluded that the main flow is nearly unaffected by the modification of the free-surface shape.

Results indicate that the release of sand, due to the aeolian erosion, occurs mainly through the upwind edge, as a result of the extended recirculation zone. The leading free-surface region is characterized during a short initial stage, by the accumulation of material, just until it reaches a stage when the free-surface's shape settles down. After that, the particle-bed, located a few millimetres above the upper-edges of the box, remains nearly unchanged. Four main zones were distinguished inside the box, depicting the sand movement inside the box.

\section{Acknowledgments}

The first author expresses his gratitude to Profs. A.C.M. Sousa and A.G. Gerber, from the University of New Brunswick-Canada, both involved in the computational simulation, for the frequent discussions and useful suggestions. Also, the great support received by the first author, during his sabbatical leave at UNB in 2007, from Prof. A.C.M. Sousa is deeply acknowledged.

The text was revised and greatly improved with the precious help of Juanita Atkins, to whom the authors extend their sincere gratitude. The construction of the perspex model was made possible due to the efforts of Mr. A.T. Cardoso.

This work was funded by FCT (Science and Technology Foundation of Portugal), (Project PTDC/EME-MFE/67631/2006) with funds from the European Community through the FEDER Program.

\section{References}

Badr, T., Harion, J.L., 2005. Numerical modeling of flow over stockpiles: implications on dust emissions. Atmos. Environ. 39, 5576-5584.

Bagnold, R.A., 1941. The Physics of Blown Sand and Desert Dunes. Chapman \& Hall, London, 265p.

Beyers, J.H.M., Sundsbø, P.A., Harms, T.M., 2004. Numerical simulation of threedimensional, transient snow drifting around a cube. J. Wind Eng. Ind. Aerod. 92, 725-747.

Ferreira, A.D., Vaz, P.A., 2004. Wind tunnel study of coal dust release from train wagons. J. Wind Eng. Ind. Aerod. 92, 565-577.

Ferreira, A.D., Viegas, D.X., Sousa, A.C.M., 2003. Full-scale measurements for evaluation of coal dust release from train wagons with two different shelter covers. J. Wind Eng. Ind. Aerod. 91, 1271-1283.

Hort, M.C., Robins, A.G., 2002. The dispersion of fugitive emissions from storage tanks. J. Wind Eng. Ind. Aerod. 90, 1321-1348. 
Ji, S.B., Gerber, A.G., Sousa, A.C.M., 2004. A convection-diffusion CFD model for aeolian particle transport. Int. J. Numer. Meth. Fluids 45, 797-817.

Lee, S.-J., Park, K.-C., Park, C.-W., 2002. Wind tunnel observations about the shelter effect of porous fences on the sand particle movements. Atmos. Environ. 36, 1453-1463.

Liu, X., Dong, Z., Wang, X., 2006. Wind tunnel modeling and measurements of the flux of wind-blown sand. J. Arid Environ. 66, 657-672.

López, M.V., 1998. Wind erosion in agricultural soils: an example of limited supply of particles available for erosion. Catena $33,17-28$.

Mansour, R.B., 2004. CFD-aided design of environmentally-friendly open-container vehicles transporting dusty materials. In: Proceedings of the IMEC2004
International Mechanical Engineering Conference, Kuwait, December 5-8, paper IMEC04-3059, 20p.

Nalpanis, P., Hunt, J.C.R., Barrett, C.F., 1993. Saltating particles over flat beds. J. Fluid Mech. 251, 661-686.

Nishimura, K., Hunt, J.C.R., 2000. Saltation and incipient suspension above a flat particle bed below a turbulent boundary layer. J. Fluid Mech. 417, 77-102.

Owen, P.R., 1964. Saltation of uniform grains in air. J. Fluid Mech. 20, 225-242.

Silva, M.C.G., Pereira, C.A.C., Cruz, J.M.S., 2003. On the use of a linear interpolation method in the measurement procedure of a seven-hole pressure probe. Exp. Therm. Fluid Sci. 28, 1-8. 